\title{
Influence of mapping complexity on negative priming for incompatible spatial mappings
}

\author{
Lenore E. Read And Robert W. Proctor \\ Purdue University, West Lafayette, Indiana
}

\begin{abstract}
For tasks with an incompatible stimulus-response mapping, whether the compatible response must be inhibited is an ongoing issue. Read and Proctor (2004) tested this inhibition hypothesis, using a negative priming paradigm for four-choice tasks with three different incompatible spatial mappings. For a mapping that did not follow a simple rule, reaction time was lengthened when the corresponding response on the preceding trial became the required response on the current trial, as compared with when it did not, showing a negative priming effect. However, for mappings that followed a simple rule, negative priming was not evident. The present study extends this research to a more complex mapping. On the basis of a two-process model adopted from the negative priming literature, we hypothesized that high mapping complexity should also diminish the negative priming effect for incompatible mappings, because the balance of cognitive resources is allocated to identification of the correct response. Two experiments are reported in which mappings of different complexity were used in six-choice spatial tasks. Analyses of reaction times showed that negative priming diminished with increased mapping complexity, apparently due to increased dominance of response identification processes, rather than inhibition of the corresponding response.
\end{abstract}

Since its inception, negative priming (NP) has been considered a useful tool for understanding the role of inhibition in control of action (Fox, 1995). In an NP task, an irrelevant stimulus or dimension on the previous trial (prime) becomes relevant on the current trial (probe). Reaction time (RT) for these trials is lengthened, as compared with trials on which the prime and probe are unrelated. This lengthening is most often attributed to residual inhibition of the irrelevant information on the prime impeding activation of the correct response to the probe (Tipper, 1985). NP has been demonstrated in many tasks, including object identity, letter identity, and semantic associations (Fox, 1995). However, the increased variety of NP tasks has shown mixed results. Depending on task requirements, NP is modulated and even reversed, and alternative accounts have been proposed that do not require inhibition, instead emphasizing retrieval (e.g., Neill, Valdes, Terry, \& Gorfein, 1992).

Recently, NP has been demonstrated in stimulusresponse compatibility (SRC) tasks. SRC refers to the finding that responses are faster when stimuli and their assigned responses correspond than when they do not. This phenomenon has been widely used to examine issues of response activation and selection in numerous task environments, as well as for various participant populations (see Proctor \& Vu, 2006). However, as with NP tasks, results in SRC tasks are subject to alternative interpretations (Read \& Proctor, 2004; Shiu \& Kornblum, 1996).
One major question is whether inhibition of the corresponding response is necessary for response selection when the stimulus-response mapping is incompatible. Incompatible mappings necessitate that participants adopt spatial transformations requiring use of short-term, taskdefined stimulus-response associations that are different from the established long-term associations between stimuli and their corresponding responses. Consequently, participants may need to inhibit the tendency to make the corresponding response activated through the long-term associations (Kornblum, Hasbroucq, \& Osman, 1990). An advantage of the NP paradigm for studying SRC effects is that it provides a measure of residual inhibition of the corresponding response. However, as was indicated, NP in both basic NP tasks and SRC tasks can be dominated by the memory requirements of the task (e.g., Chao \& Yeh, 2008; Proctor, Vu, \& Marble, 2003).

\section{A Two-Process Model for SRC}

Two-process models have been proposed for both SRC and NP. In the compatibility literature, Kornblum et al.'s (1990) dimensional overlap model is among the most popular. According to it, when stimulus and response sets have conceptual or perceptual correspondence, the corresponding response is automatically activated. The assigned response is also identified via a response identification process. If the automatically activated corre-

L.E.Read,readl@psych.purdue.edu 
sponding response matches the identified response, it is executed quickly. However, if the identified response is different, the corresponding response must be inhibited before the correct response can be initiated. Shiu and Kornblum (1996) demonstrated NP in a symbolic task. Pictures of four objects were alternated with the printed words that represent them, with both types of stimuli mapped incompatibly to the same words as vocal responses (e.g., car $\rightarrow$ "bike"; bike $\rightarrow$ "boat"; boat $\rightarrow$ "plane"; plane $\rightarrow$ "car"). NP was defined as a longer RT when the stimulus identity on the prime trial became the probe response (e.g., car $\rightarrow$ "bike," followed by plane $\rightarrow$ "car"), as compared with trials on which the prime and probe were unrelated. Shiu and Kornblum interpreted this NP as reflecting residual inhibition of the corresponding response from the prime trial. Furthermore, they suggested that this inhibition occurs at the executive level and is nonspecific to the task.

Contrary to Kornblum et al.'s (1990) model, which specifies that the corresponding response is activated and must be inhibited for all incompatible mappings, Read and Proctor (2004) found that NP in four-choice spatial tasks depended on the complexity of the stimulus-response mapping. NP was observed when the mapping did not follow a simple rule (Experiment 1), as in Shiu and Kornblum's (1996) study. However, when a simple rule could be implemented (e.g., respond "mirror opposite"; Experiment 2), NP disappeared and reversed to positive priming (a benefit when prime and probe trials were related).

Although robust, as with NP, compatibility effects can be reduced or reversed by particular task demands (Proctor et al., 2003; Stoffels, 1996). Duncan (1977) was the first to suggest that compatibility effects can depend on the task-defined transformational rules. By examining error types in a series of four-choice tasks, he demonstrated conditions under which transformational rules took precedence over individual stimulus-response associations, thus decreasing the compatibility effect.

Marble and Proctor (2000) mixed location-relevant with location-irrelevant trials of a Simon task (Simon, 1990), for which RT is typically lengthened for trials on which the stimulus-response relation is spatially incompatible. When the location-relevant task mapping was incompatible, a reverse Simon effect occurred, favoring incompatible spatial relations. Marble and Proctor suggested that the spatial mapping defined for the location-relevant task determined the Simon effect. Proctor et al. (2003) extended these findings with two location-relevant tasks. Their results implied that under some conditions in which a location code can provide a basis for responding, a task set can be established that overrides the normal compatibility effect.

Recently, in the NP literature, there have been various attempts to explain the differences in NP between studies and the role of inhibition. These explanations suggest that $\mathrm{NP}$ as a reflection of inhibition depends on the balance of other processes involved in a particular task. For example, Kossowska (2007) showed that cognitive load can affect the strength of NP. According to this view, inhibition is part of a general resource pool. When cognitive load is increased, fewer resources are available for inhibition and, hence, the NP effect decreases. Chao and Yeh (2008) suggested a two-process model in which modulation of the NP effect depends on a balance of attentional inhibition and memory retrieval. In the case of NP, the contributions of probe distractor strength, or salience, and prime-probe similarity determine the dominant influence of inhibition or retrieval, respectively. Marczinski, Milliken, and Nelson (2003) argued that NP reflects the joint influence of two processes, which they called specific and nonspecific. Specific influence depends on the mapping required by the task, and nonspecific influence "depends on the prior response independent of the stimulus to which it was made" (p. 788).

These NP studies offer a perspective for viewing modulation of SRC effects. It is known that SRC effects can depend on task demands. A two-process model that includes both specific (i.e., identification of the task-defined response) and nonspecific (i.e., inhibition of the corresponding response) factors, and the subsequent domination of either of these processes, can offer a possible explanation of why some experiments implicate transformational processes involved in response identification (e.g., Duncan, 1977; Read \& Proctor, 2004) but others suggest inhibition of the corresponding response (e.g., Shiu \& Kornblum, 1996). Following the premise that participants choose the most salient route from perception to action, an important question is whether there are conditions under which response identification takes precedence over inhibition of the corresponding response. Read and Proctor (2004) demonstrated that when the task mapping follows an easily defined rule, NP is reversed, arguing against the necessity of inhibiting the corresponding response. In contrast, recent evidence in the NP literature suggests that NP, as a reflection of the inhibitory aspects of attentional control, diminishes when tasks are extremely demanding (Kossowska, 2007). We examined whether this principle applies to spatial compatibility tasks.

The following two experiments tested whether increasing cognitive load by increasing task complexity would diminish the NP effect, using a six-choice spatial task. The basic difference between the experiments was the complexity of the mapping. On the basis of the concept of a two-process model, as suggested by the NP literature, our underlying assumption was that incompatible stimulus-response mappings involve both general (control of interference by inhibiting the corresponding response) and specific (identification of the assigned response) processes. As mapping complexity increases, response identification dominates attentional resources. Another way of stating this is that identification of the assigned response becomes more salient and the balance of attention favors this specific process. In turn, working memory becomes more occupied with specific processes, and fewer resources are allocated to general processes of cognitive control (e.g., Engle, Conway, Tuholski, \& Shisler, 1995). The corresponding response is less salient and less intrusive on attentional resources, and attention is occupied with task mapping. Hence, NP should diminish. 


\section{THE EXPERIMENTS}

Experiments 1 and 2 used different mappings for a sixchoice incompatible spatial task to examine whether an increase in mapping complexity would increase specific task load and cause allocation of attentional resources to response identification to dominate, thus making interference from the corresponding response less dominant. The mapping for Experiment 1 (Figure 1A) was similar, in terms of consistency of the stimulus-response mapping, to that for the four-choice task in Read and Proctor's (2004) Experiment 1 but was applied to a six-choice task. Because that type of mapping showed NP in a four-choice task, we expected it also to show NP in the present Experiment 1. The Experiment 2 mapping (Figure 1B) was made more complex by employing an inconsistent mapping pattern, accomplished by reversing the response pairings for the inner positions to each side of the focus point. Past research has shown that inconsistent mappings yield longer RTs than do consistent mappings, suggesting greater response identification difficulty (e.g., Duncan, 1977). Due to this greater mapping complexity, we expected that RT would be lengthened, and, more important, NP diminished, as compared with Experiment 1. In past research, cognitive load has been varied not by increasing response identification difficulty but through perceptual manipulations, individual differences in working memory, or introduction of a secondary task, for example. This is the first

\section{A}

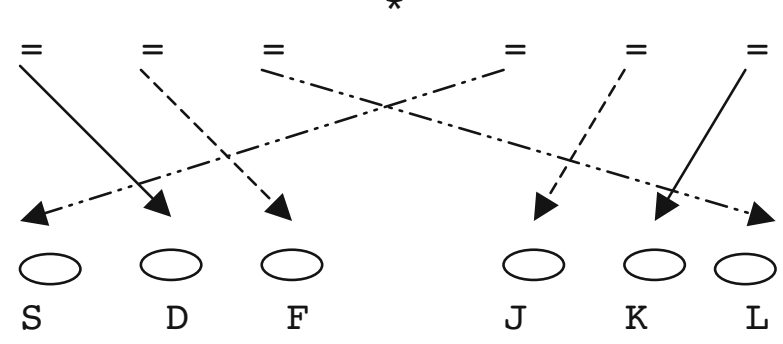

B

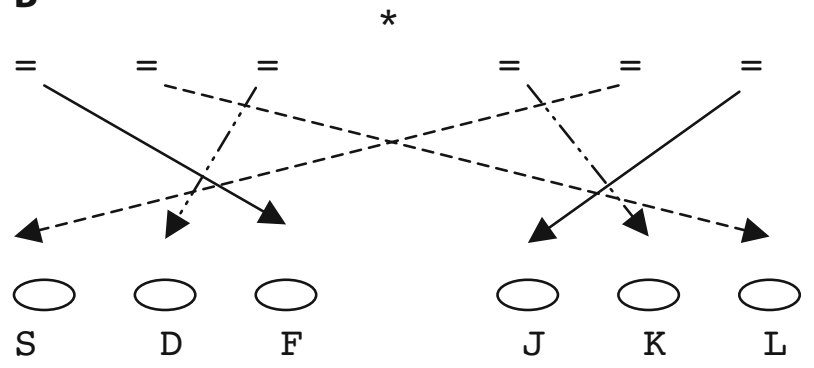

Figure 1. Stimulus-response mappings for Experiment 1 (A) and Experiment 2 (B). Participants responded by pressing the $S, D, F, J, K$, or $L$ key, using their index, middle, and ring fingers of each hand in their natural position. In the example of the $S-C R$ condition in panel $A$, the stimulus location for the prime response $D$ became the response for the probe $S$; in panel $\mathrm{B}$, the stimulus location for the prime $F$ became the response for probe $S$. For neutral trials, there was no relation between the locations of the target and the responses on the prime and probe trials. study in which cognitive load was increased by concentrating on the task demands of the process in question.

\section{Method}

Participants. Sixty participants (30 in each experiment) were recruited from introductory psychology courses for course credit.

Stimuli and Apparatus. Stimuli were presented on a 14-in. VGA color monitor of a PC. Location markers were six equal signs on the 11 th print line. An asterisk acted as a focus point in the center of the 10th print line. The target was the letter $X\left(0.64 \mathrm{~cm} / 0.67^{\circ}\right.$ high and $0.32 \mathrm{~cm} / 0.33^{\circ}$ wide), presented in white on the 10 th print line, directly above the location markers. The combined height of the target letter and location marker was $0.95 \mathrm{~cm} / 0.99^{\circ}$. The stimuli and fixation point were separated by three print spaces $\left(0.96 \mathrm{~cm} / 1.0^{\circ}\right)$. The participants responded by pressing the $S, D$, or $F$, and $J, K$, or $L$ keys of a standard computer keyboard, which were marked blue to avoid any association between the letters and the stimulus location.

Procedure. Apart from the mapping (Figure 1), Experiments 1 and 2 were exactly the same. The participants sat $55-60 \mathrm{~cm}$ from the computer screen. They received task instructions that emphasized accuracy and speed equally. Trial types were adapted to coincide with the experiments in Read and Proctor (2009) in which the effects of the previous prime-probe interval as a marker for episodic retrieval were considered (Neill et al., 1992). Therefore, trials were grouped into triplets. Each triplet began with a fixation point, “*," and six location markers for $1,000 \mathrm{msec}$ before the target's appearance for trial $l_{n}$. The response for trial $n$ was followed by a responsestimulus interval (RSI) of 500, 1,100, or 2,000 msec before the appearance of trial ${ }_{n+1}$. Trial ${ }_{n+1}$ was then followed by a constant RSI of $500 \mathrm{msec}$ followed by trial ${ }_{n+2}$. Trial types for trial ${ }_{n+1}$ and trial ${ }_{n+2}$ were either neutral or stimulus-correct response (S-CR) transitions, as used in previous experiments (Read \& Proctor, 2004). For S-CR trial events, the previous trial's stimulus location (and, hence, the corresponding response) became the present trial's correct response location. Combinations of trial type transitions for trial ${ }_{n+1}$ to trial $l_{n+2}$ consisted of neutral to S-CR, S-CR to neutral, or neutral to neutral, creating a 2 to 1 ratio of neutral-to-S-CR trial types for both trial $l_{n+1}$ and $\operatorname{trial}_{n+2}$.

The participants performed 20 practice trial triplets, followed by two blocks of test trials. For test blocks, order of RSI between trial $_{n+1}$ and $\operatorname{trial}_{n+2}$ was randomized within blocks, and each trial combination appeared only once for each RSI, resulting in 108 trial triplets per block. Feedback for errors and correct RTs was given during practice, and only feedback for errors was given during test trials.

\section{Results: Experiment 1}

To assure attentiveness during trial $_{n+1}$ and trial $_{n+2}$, only trial types with correct responses for each trial event were included in the RT analyses. Frequency distributions of overall correct RTs for trial ${ }_{n+1}$ and trial ${ }_{n+2}$ were computed separately, and $2 \%$ of the extreme scores were eliminated as outliers. The resultant RT distributions included approximately three standard deviations from the resulting mean. Mean RT for trial ${ }_{n}$ was $857 \mathrm{msec}(S D=183)$. Error rate $(\mathrm{ER})$ was $1.5 \%(S D=1.1 \%)$.

An ANOVA for RT for trial ${ }_{n+1}$, with RSI and trial type as within-subjects factors, resulted only in a significant main effect of trial type $\left[F(1,29)=9.74, M S_{\mathrm{e}}=1,700\right.$, $p<.005]$ : RT was $19 \mathrm{msec}$ longer for S-CR trial types $(870 \mathrm{msec})$ than for neutral trial types $(851 \mathrm{msec})$, showing NP (see Table 1). A similar ANOVA for ER also yielded a main effect for trial type $\left[F(1,29)=5.68, M S_{\mathrm{e}}=6.1\right.$, $p<.03]$ : ER was higher for S-CR $(2 \%)$ than for neutral (1\%) trial types. A further analysis of error-types was performed (Table 2), with the rationale that errors in re- 
Table 1

Reaction Times (RTs, in Milliseconds) and Error Rates (ERs, in Percentages) by Trial and Trial Type in Experiments 1 and 2

\begin{tabular}{|c|c|c|c|c|c|c|}
\hline & \multicolumn{4}{|c|}{ Trial Type } & & \\
\hline & \multicolumn{2}{|c|}{$\begin{array}{l}\text { Neutral/ } \\
\text { Control }\end{array}$} & \multicolumn{2}{|c|}{$\mathrm{S}-\mathrm{CR}$} & \multicolumn{2}{|c|}{$\begin{array}{c}\text { Priming } \\
\text { Effect }\end{array}$} \\
\hline & RT & ER & RT & ER & RT & ER \\
\hline \multicolumn{7}{|c|}{$\operatorname{Trial}_{n+1}$} \\
\hline Experiment 1 & 851 & 1 & 870 & 2 & -19 & -1 \\
\hline Experiment 2 & 1,052 & 3 & 1,052 & 3 & 0 & 0 \\
\hline \multicolumn{7}{|c|}{$\operatorname{Trial}_{n+2}$} \\
\hline Experiment 1 & 861 & 1 & 882 & 3 & -21 & -2 \\
\hline Experiment 2 & 1,049 & 2 & 1,018 & 2 & +31 & 0 \\
\hline
\end{tabular}

Note-S-CR, trials for which the stimulus on the preceding trial became the correct response on the current trial. Priming effect $=$ neutral $-\mathrm{S}-\mathrm{CR}$.

sponse reflect the processes involved in response selection. The categorization of errors was adapted from that used by Duncan (1977). For S-CR trial types, 60\% were the corresponding response, with $17 \%$ being a repetition of the previous response, $17 \%$ the correct mapping but the wrong hand, and $6 \%$ other (i.e., arbitrary finger). For neutral trial types, $47 \%$ of the errors consisted of the corresponding response.

For trial ${ }_{n+2}$, analyses included only the neutral-toneutral transitions and the neutral-to-S-CR transitions. ${ }^{1}$ The ANOVA of RT as a function of previous RSI and trial type yielded a main effect of trial type $[F(1,29)=6.15$, $\left.M S_{\mathrm{e}}=3,302, p<.02\right]$. Again, RT was longer for S-CR $(882 \mathrm{msec})$ than for neutral trial types $(861 \mathrm{msec})$. (See Table 1.) The analysis of ER also yielded only a significant effect of trial type $\left[F(1,29)=16.52, M S_{\mathrm{e}}=10.4\right.$, $p<.0003$ ], with a higher ER for S-CR (3\%) than for neutral (1\%) trial types. The results were similar to those for trial ${ }_{n+1}$, with $59 \%$ of the errors consisting of the corresponding response (Table 2).

Under the mapping conditions in Experiment 1, the results for error types suggest considerable interference from the corresponding response for both trial ${ }_{n+1}$ and trial $_{n+2}$, suggesting that, at this level of complexity, neither the more general process of inhibiting the corresponding response nor the specific processes involved in retrieval of task rules dominated.

\section{Results: Experiment 2}

Mean RT for trial ${ }_{n}$ was $1,026 \mathrm{msec}(S D=167)$. ER was $2.8 \%(S D=2.3 \%)$. Mean RT was longer and ER higher than in Experiment $1[t \mathrm{~s}(58)=3.6$ and 3.0], reflecting the added difficulty of Experiment 2.

The ANOVA for trial $n+1$ yielded a main effect for RSI only $\left[F(2,58)=3.94, M S_{\mathrm{e}}=6,493, p<.03\right]$. RT increased between $500-\mathrm{msec}(1,035 \mathrm{msec}), 1,100-\mathrm{msec}$ $(1,046 \mathrm{msec})$, and $2,000-\mathrm{msec}(1,075 \mathrm{msec})$ RSIs. The increase in RT with RSI could reflect an adjustment in response or in retrieval with the added timing. RT was the same for S-CR trial types $(1,052 \mathrm{msec})$ and neutral trial types $(1,052 \mathrm{msec})$. (See Table 1.) Univariate analysis assessing differences in NP between Experiments 1 and 2, with RSI collapsed, confirmed a significant decrease in NP for Experiment $2[F(1,58)=7.31, p<.008]$.

For ER, there were no differences between the two trial types (approximately $3 \%$ ) or by RSI. As is evident in Table 2, only a small percentage of S-CR trial types consisted of intrusions of the corresponding response. In addition, error types were similar for S-CR and neutral trial types. The proportion of errors involving the corresponding response was lower than that in Experiment 1 $\left[\chi^{2}(1)=30.5\right]$.

For trial ${ }_{n+2}$, the positive priming effect did reach significance. The analysis of RT by trial type and previous RSI yielded only a main effect of trial type $[F(1,29)=$ 9.65, $\left.M S_{\mathrm{e}}=11,032, p<.05\right]$ : RT was shorter for S-CR trial types $(1,018 \mathrm{msec})$ than for neutral trial types $(1,049 \mathrm{msec}$ ). (See Table 1.) An ANOVA between NP for Experiments 1 and 2 again confirmed the decrease in NP for Experiment $2[F(1,58)=4.40, p<.001]$.

The ANOVA for ER was nonsignificant (mean ER = $2 \%$ ). Both S-CR and neutral trial types for $\operatorname{trial}_{n+2}$ were similar to those for $\operatorname{trial}_{n+1}$ (see Table 2), showing only a small percentage of intrusions of the corresponding response (14\%). Again, differences in the proportion of errors for S-CR trial types involving the corresponding response were lower than in Experiment $1\left[\chi^{2}(1)=49.9\right]$. In both Experiments 1 and 2, large proportions of other errors $(30 \%-50 \%)$ were mirror-opposite responses to the stimulus, suggesting that the saliency of this relation is such as to interfere even when not part of the task mapping.

\section{Discussion}

In the present study, we examined processes in spatial compatibility tasks under increased mapping complexity.

Table 2

Percentages of Error Types for $\operatorname{Trial}_{n+1}$ and $\operatorname{Trial}_{n+2}$ in Experiments 1 and 2

\begin{tabular}{|c|c|c|c|c|}
\hline & $\begin{array}{c}\text { Corresponding } \\
\text { Response }\end{array}$ & $\begin{array}{l}\text { Previous } \\
\text { Response }\end{array}$ & $\begin{array}{l}\text { Wrong } \\
\text { Hand }\end{array}$ & Other \\
\hline \multicolumn{5}{|c|}{ S-CR Trial Type } \\
\hline \multicolumn{5}{|l|}{$\operatorname{Trial}_{n+1}$} \\
\hline Experiment 1 & 60 & 17 & 17 & 6 \\
\hline Experiment 2 & 14 & 7 & 22 & 57 \\
\hline \multicolumn{5}{|l|}{$\operatorname{Trial}_{n+2}$} \\
\hline Experiment 1 & 59 & 5 & 11 & 25 \\
\hline Experiment 2 & 14 & 8 & 22 & 56 \\
\hline \multicolumn{5}{|c|}{ Neutral Trial Type } \\
\hline \multicolumn{5}{|l|}{$\operatorname{Trial}_{n+1}$} \\
\hline Experiment 1 & 47 & 2 & 15 & 36 \\
\hline Experiment 2 & 16 & 9 & 10 & 62 \\
\hline \multicolumn{5}{|l|}{$\operatorname{Trial}_{n+2}$} \\
\hline Experiment 1 & 49 & 6 & 16 & 28 \\
\hline Experiment 2 & 15 & 4 & 11 & 71 \\
\hline
\end{tabular}

Note-Percentage of errors was computed as the number of errors in that category divided by the total number of errors. S-CR, trials for which the stimulus on the preceding trial becomes the correct response on the current trial; corresponding response, corresponding response on present trial; previous response, repetition of response used on previous trial; wrong hand, correct mapping but wrong hand; other, errors that were neither the corresponding response nor systematic in terms of the spatial transformations used. No errors were the previous corresponding response. 
The results suggest that as mapping complexity increases, the balance of attentional resources favors response identification as the more salient route toward action selection, resulting in less interference from the corresponding response. In Experiment 2, RT was longer and error greater for the S-CR trial types than in Experiment 1, reflecting the increased difficulty of Experiment 2 mapping. Whereas error types in Experiment 1 were equally split between intrusions of the corresponding response and other errors, error types in Experiment 2 reflected a domination of errors that did not involve the corresponding response.

A two-process model. Our approach was adopted from a two-process model originally formulated for NP effects (Marzcinski et al., 2003). According to this view, the process that contributes to NP is effortful and resource dependent. It is not a pure inhibitory process, and it shares attentional resources with selectivity and memory retrieval. We suggest that task demands determine whether attention is dominated by the general processes of response inhibition or the more specific processes entailing response identification. That is, when the incompatible mapping is sufficiently complex, participants adopt a task set that overrides the normal compatibility effect (Proctor et al., 2003). In other words, modulation of NP reflects the balance of attentional resources that are allocated to the stimulus-response mapping. Greater allocation leads to less NP because the corresponding response is a less salient and, hence, a less intrusive aspect of the experimental condition. In keeping with this hypothesis, RT increased and NP was reduced for the more complex mapping in Experiment 2, as compared with Experiment 1.

Results showing that NP occurs only for certain incompatible mappings challenge dual-route models in which the corresponding response is always activated and, therefore, must always be inhibited (e.g., Kornblum et al., 1990). If the corresponding response is activated to a similar extent regardless of mapping, the absence of NP suggests that inhibition of that activation is not necessary when the mapping is very complex or that the effect of prime trial inhibition on the probe trial is ameliorated by the mapping difficulty. A final alternative is that increased focus on response identification reduces activation of the corresponding response on all trials. The positive priming in Experiment 2 could be explained in terms of lack of distractor (i.e., the corresponding response) interference (Neill \& Joordens, 2002) on the probe trial. In any case, with increased task complexity, the corresponding response was less dominant.

The results of Experiments 1 and 2 and those of Read and Proctor (2004) have shown two instances whereby response identification takes precedence over the corresponding response: when the mapping is simple or when it is complex. The explanation we favor is that these instances are due to the increased salience of response identification, but for two different reasons. Duncan (1977) showed that an opposite mapping rule yields shorter RT than do other inconsistent mappings. This was the case with Experiments 2 and 3 in Read and Proctor (2004). Duncan also showed longer RT for inconsistent mappings.
In his manipulation, a key finding was through analysis of error types to infer the use of transformational rules. In the present study, the separation of trial types aids in defining the balance of attentional resources between specific task-based mapping and intrusion of the corresponding response.

The advantage with the respond opposite mapping in Read and Proctor's (2004) study is that it creates a reversal between stimulus-response codes. This can best be explained by the common coding viewpoint of perception to action (Prinz, 1990). Accordingly, stimuli and response codes share the same representational medium, with response selection rules requiring a separate linkage. It can be argued that previous activation of the prime stimulus position and its linkage with the probe stimulus position aided its subsequent use on the probe trial.

For the more difficult mapping used in the present Experiment 2 , however, the balance of attentional resources was dominated or captured by the processes involved in identification of the specific response, resulting in less interference from the corresponding response. The results suggest that the saliency and, hence, dominance of response identification processes apply either when participants can adopt a simple transformational rule or when the mapping is sufficiently difficult to tax attentional resources. This pattern suggests that attentional allocation is influenced by the saliency of the stimulus-response transformations for a task set.

Interpretations of SRC and NP effects are often eitheror. A two-process model that considers the balance of attentional control and retrieval of task mappings may be a better evaluation of effects. In the SRC literature, it raises challenges to current dual-process models and can lead to a greater understanding of processes in action selection.

\section{AUTHOR NOTE}

The research described in the present article was supported in part by NIA Grant R01 G021071. We thank Tiffany Forte for help with data collection. Correspondence concerning this article should be addressed to L. E. Read, Department of Psychological Sciences, Purdue University, 703 Third St., West Lafayette, IN 47907-2081 (e-mail: readl@psych .purdue.edu)

\section{REFERENCES}

Chao, H.-F., \& Yeh, Y.-Y. (2008). Attentional demand and memory retrieval in negative priming. Psychological Research, 72, 249-260. doi:10.1007/s00426-006-0106-y

Duncan, J. (1977). Response selection rules in spatial choice reaction tasks. In S. Dornič (Ed.), Attention and performance VI (pp. 49-61). Hillsdale, NJ: Erlbaum.

Engle, R. W., Conway, A. R. A., Tuholski, S. W., \& Shisler, R. J. (1995). A resource account of inhibition. Psychological Science, 6, 122-125. doi:10.1111/j.1467-9280.1995.tb00318.x

Fox, E. (1995). Negative priming from ignored distractors in visual selection: A review. Psychonomic Bulletin \& Review, 2, 145-173.

Kornblum, S., Hasbroucq, T., \& Osman, A. (1990). Dimensional overlap: Cognitive basis for stimulus-response compatibility-A model and taxonomy. Psychological Review, 97, 253-270. doi:10.1037/0033 $-295 X .97 .2 .253$

KossowskA, M. (2007). The role of cognitive load and memory capacity in negative priming. Polish Psychological Bulletin, 38, 15-23.

Marble, J. G., \& Proctor, R. W. (2000). Mixing location-relevant and location irrelevant choice-reaction tasks: Influences of location mapping on the Simon effect. Journal of Experimental Psychology: 
Human Perception \& Performance, 26, 1515-1533. doi:10.1037/0096 $-1523.26 .5 .1515$

Marczinski, C. A., Milliken, B., \& Nelson, S. (2003). Aging and repetition effects: Separate specific and nonspecific influences. Psychology \& Aging, 18, 780-790. doi:10.1037/0882-7974.18.4.780

Neill, W. T., \& Joordens, S. (2002). Negative priming and multiple repetition: A reply to Grison and Strayer (2001). Perception \& Psychophysics, 64, 855-660.

Neill, W. T., Valdes, L., Terry, K., \& Gorfein, D. (1992). Persistence of negative priming: II. Evidence for episodic trace retrieval. Journal of Experimental Psychology: Learning, Memory, \& Cognition, 18, 993-1000. doi:10.1037/0278-7393.18.5.993

PRINZ, W. (1990). A common coding approach to perception and action. In O. Neumann \& W. Prinz (Eds.), Relationships between perception and action: Current approaches (pp. 167-201). Berlin: Springer.

Proctor, R. W., \& Vu, K.-P. L. (2006). Stimulus-response compatibility principles: Data, theory, and application. Boca Raton, FL: CRC Press.

Proctor, R. W., Vu, K.-P. L., \& Marble, J. G. (2003). Spatial compatibility effects are eliminated when intermixed location-irrelevant trials produce the same spatial codes. Visual Cognition, 10, 15-50. doi: $10.1080 / 713756673$

ReAD, L. E., \& Proctor, R. W. (2004). Spatial stimulus-response compatibility and negative priming. Psychonomic Bulletin \& Review, 11, 41-48.
Read, L. E., \& Proctor, R. W. (2009). Stimulus-response compatibility and negative priming in symbolic and spatial tasks. Manuscript in preparation.

ShiU, L.-P., \& Kornblum, S. (1996). Negative priming and stimulusresponse compatibility. Psychonomic Bulletin \& Review, 3, 510-514.

Simon, J. R. (1990). The effects of an irrelevant directional cue on human information processing. In R. W. Proctor \& T. G. Reeve (Eds.), Stimulus-response compatibility: An integrated perspective (pp. 3186). Amsterdam: North-Holland.

StOFFELS, E. J. (1996). Uncertainty in processing routes in the selection of a response: An S-R compatibility study. Acta Psychologica, 94, 227-252. doi:10.1016/0001-6918(95)00063-1

TIPPER, S. P. (1985). The negative priming effect: Inhibitory priming by ignored objects. Quarterly Journal of Experimental Psychology, 37A, 571-590.

\section{NOTE}

1. S-CR-to-neutral transitions were excluded for both experiments to avoid possible carryover effects to trial ${ }_{n+2}$. Hence, for the analyses, there was a $1: 1$ ratio of neutral to $\mathrm{S}-\mathrm{CR}$ trial types.

(Manuscript received June 18, 2008; revision accepted for publication July 3,2009 .) 